\title{
Exploration and Reflections of Multi Mobile Internet Technology in Training Management
}

\author{
Guoxing Han \\ Development Research Center, Transport Management Institute Ministry of Transport, Beijing, China
}

\section{Email address:}

hanguoxing@motmti.cn

\section{To cite this article:}

Guoxing Han. Exploration and Reflections of Multi Mobile Internet Technology in Training Management. Science Innovation. Vol. 7, No. 6, 2019, pp. 149-156. doi: 10.11648/j.si.20190706.11

Received: November 4, 2019; Accepted: December 4, 2019; Published: December 9, 2019

\begin{abstract}
This paper ,firstly, combs the basic process and existing problems of current training management, based on the actual and present facts; Secondly, the application of questionnaire platform, online collaboration documents and other tools to improve the training management process is proposed to explore the practice with the current mobile Internet technology, to improve the efficiency of training management and reduce the workload of managers. Finally, considering the convenience of mobile Internet technology and the feasibility of wechat public platform application in education and training, the construction of wechat public platform training management section of development research center is considered.
\end{abstract}

Keywords: Mobile Internet Technology, Questionnaire Platform, Collaborative Documents, Wechat, Training Management

\section{多样化移动互联网技术在培训管理中的应用探索与思考}

\section{韩国兴}

交通运输部管理干部学院发展研究中心, 北京, 中国

邮箱

hanguoxing@motmti.cn

摘要: 文章首先从我院发展研究中心自主班次的培训管理工作实际出发, 梳理当前培训管理的基本流程及其存在的问 题。其次，基于当前移动互联网技术，提出了应用问卷平台、在线协作文档等工具改进培训管理过程的探索实践，从 一定程度上提升培训管理的工作效率、减轻管理人员的工作量。最后, 综合考虑移动互联网技术的便捷性及微信公众 平台在教育培训中应用的可行性, 对发展研究中心开展微信公众平台培训管理板块的建设进行了思考。

关键词: 移动互联网技术, 问卷平台, 协作文档, 微信公众平台, 培训管理

\section{1. 引言}

培训管理工作是一项重复且繁琐的工作，培训管理教 师作为教育培训活动的组织者和管理者, 要投入相当大的 时间和精力, 确保培训工作的有序、精准和高效进行。然 而, 当前培训管理中招生、登记、实施、评估等诸多环节
的工作事项琐碎、单调重复, 占据培训管理人员大量的时 间和精力。

如何有效提升发展研究中心（简称中心）自主班次的 培训管理工作, 加强与学员在培训不同阶段的沟通交流, 提升工作效率、减少时间和精力的消耗, 同时又能增强学 员对中心教育培训效果和培训品牌的认可, 是我们不容忽 视的问题。 
随着培训业务量逐渐增大, 传统的管理方式从技术上、 效率上都难以适应发展需要, 干部教育学院急需有一款适 合干部培训工作的信息管理系统。为此, 许多学者和专家 对干部培训管理信息系统进行了诸多实践探索。洪东 [1] 以某事业单位的干部培训管理需求为背景, 利用现化信息 技术建立干 部培训管理系统, 是为了推进干部培训管理 的信息化建设, 提高干部培训管理 水平和行政工作效率。 李雪玲 [2]通过构建基于 $\mathrm{C} / \mathrm{S}$ 模式的培训管理信息系统, 系 统实现了培训管理 各阶段数据信息化、日常管理事务轻 松化、工作人员少量化。无疑, 信息化系统的建设和完善, 能够有效提升干部教育培训的效率和管理水平, 但由于信 息化系统的建设成本和建设周期, 以及干部培训管理系统 的普适性不强等问题, 使得培训管理系统功能难以一致, 可复制性差。

随着互联网技术的不断更新和发展, 轻量化、便捷化、 经济化、普适化的管理辅助手段得到广大用户的青睐, 利 用移动互联网技术辅助现代工作已成为新的趋势。如 2012 年中国银联培训中心开通微信公众号, 实现需求调研、培 训评估、培训咨询、移动学习、实时交互、班级管理等功 能。2016年, 罗庞塬采油作业区利用微信和问卷星开展“问 卷星微信答题”。因此, 针对如何借助多样化的移动互联 网技术中心的培训管理工作, 提高工作效率, 中心开展了 探索和实践并取得了一定成果。

\section{2. 中心自主班次培训管理流程及其问题分析}

自主培训班次主要是区别于每年的部办计划培训班 次的发问培训项目。培训管理的流程集中反映了培训组织 的培训管理理念, 体现培训机构在培训管理实践中的能力 与水平。运用流程管理的思想方法和工具、路径, 在培训 管理中加强流程建设, 可以提高培训管理效率与水平。

\section{1. 自主班次培训管理流程}

间琳[3]认为从培训班准备到结束, 需要完成 33 个培训 管理流程项目, 包括16项训前工作、13项训中工作及4项 训后工作（表1）。

表1 培训管理流程项目。

\begin{tabular}{ll}
\hline 阶段 & 内容 \\
\hline 训前16项 & 培训需求分析、设计班型、编制上报培训班通知、编制 \\
& 学员手册、安排总课表、编制培训班经费预算等 \\
& 开班仪式、入学教育相关工作、组建班委会、授课组织 \\
训中13项 & 相关工作、教学参观和考察相关工作、课外活动组织工 \\
& 作等 \\
训后4项 & 撰写培训班总结、编制培训班决算、整理并上交培训班 \\
& 档案 \\
\hline
\end{tabular}

通过对自主培训班次培训管理过程的回顾和归纳, 简 单将其流程归纳如错误!未找到引用源。所示的训前、训 中、训后三个阶段:

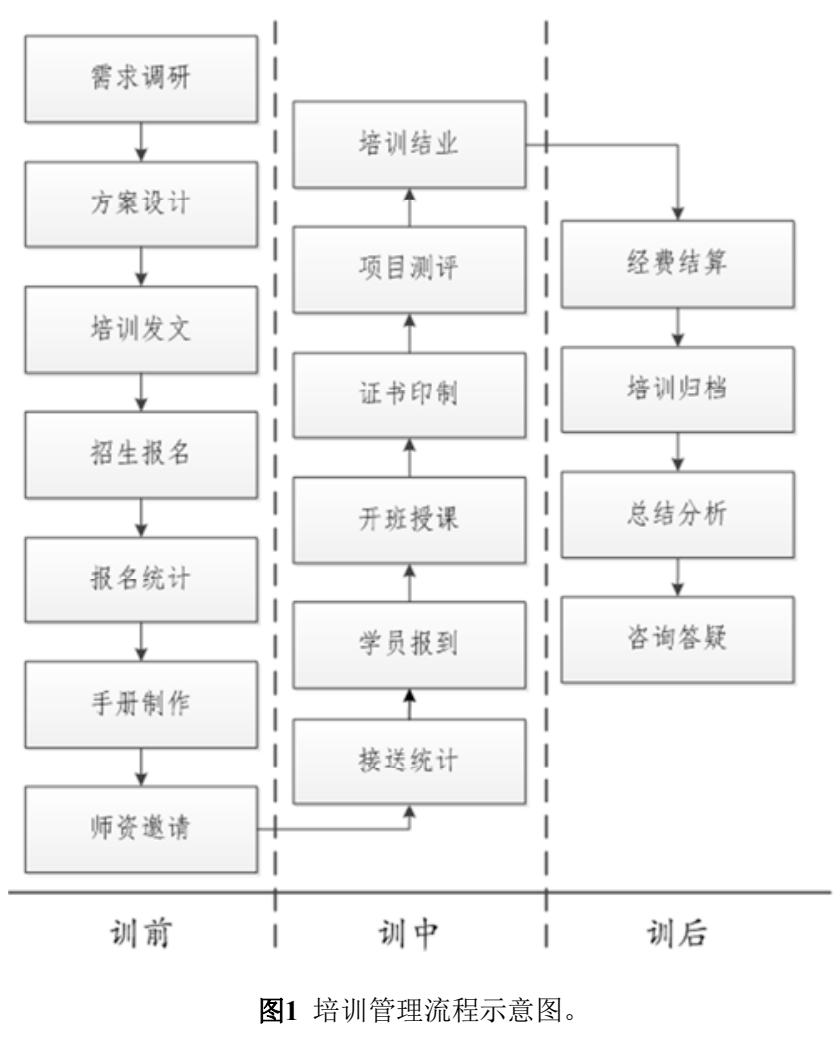

\section{2. 培训管理流程中存在的问题}

培训项目的管理流程中存在的问题主要存在培训报 名统计、学员报到、印制证书。

\subsection{1. 报名统计}

传统的培训管理过程中, 培训项目的发文一般通过邮 寄、传真或微信、QQ（群）等方式发到相关单位, 但是 范围十分局限。而且参训人员报名通常根据通知文件, 以 传真文件或电子文件形式报名, 因此在报名统计过程中学 员信息录入和登记工作贯穿发文后到报到前的过程, 耗时 长、费力多, 且随着报到日期的临近报名数量剧增骤降 (如 图2、图3）。

\subsection{2. 学员报到}

在学院信息化系统建成并投入使用之前, 学员报到多 数仍将采用现场签字签到的方式。一般签到前参训学员仅 能通过短信、邮件或传真等方式获取部分报到和培训安排 等信息, 获取信息量在一定程度受到局限。由于培训期间 还需要学员核对单位、职务及联系方式等重要个人信息, 若学员报到时当场签到并核对信息虽然方便, 但难免存在 重要信息易泄露的风险, 且在下午集中报到时间段内签到 核对效率偏低。 


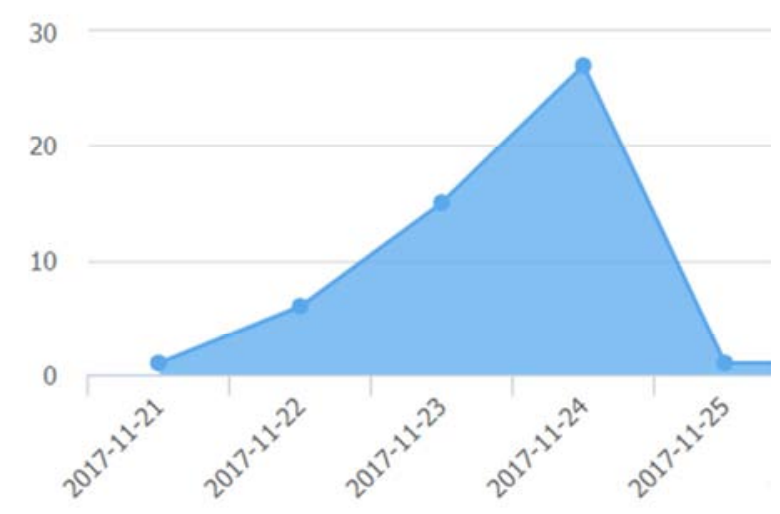

图2 北京市委托某培训班的报名数量变化趋势图。

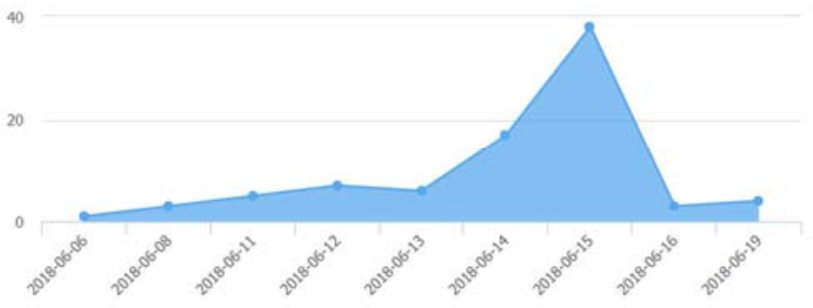

图3 一卡通某政策宣贯会的报名数量变化趋势图。

\subsection{3. 证书印制}

学员证书制作的过程首先通过学员提交电子版照片, 其次根据培训科研处要求的格式更改照片备注名、填写证 书申领资料表, 最后由科研培训处批量印制。影响证书印 制工作效率的工作量主要集中在: (1)证书材料表中 (学员) 编号姓名字符长度的调整; (2)证书材料表中 (学员) 照片 文件名的生成; (3)与证书材料表中 (学员) 照片文件名对 应的学员照片文件名的修改。其中, 对培训组织管理效率 产生较大影响的是同步修改证件照片文件名的字符长度 并添加学员序号。当培训班学员数量较少时, 该工作可以 通过人工重复重命名照片文件完成, 但是随着培训班学员 数量的增加, 该项工作耗时和繁琐程度将会十分明显。

上述问题在培训组织管理过程中对管理效率的影响 较大。除此之外, 培训课程和项目的评估过程中的问卷发 放、收集分析工作也有待改进。

\section{3. 中心关于多样化移动互联网技术在培训管理 中的应用探索}

随着宽带无线移动通信技术的进一步发展和Web应 用技术的不断创新, 移动互联网的技术呈现出了多样化的 趋势 [4]。其中, 微信、问卷平台及在线协作文档工具等新 技术的产生和发展, 对提升工作和管理效率给予了很大的 辅助。

\section{1. 移动互联网技术在培训管理中的应用}

近年来, 一些专家学者对移动互联网技术在教育培训 中的应用的可行性及运用策略开展了不少研究工作。郭伟
才[5]认为通过微信群的沟通、联谊和空间感能够更好地实 现学员管理的实时化, 公众平台的开发有助于培训管理的 虚拟化、移动化和实时化, 同时通过微信平台的数据积累, 进而建立移动数据库都有助于培训中动态资源的集聚, 使 精准管理、精准营销成为可能, 并大幅降低实现成本。余 雅斌[6]从教师培训存在的现实问题出发, 探索了微信及其 公众平台在教师培训中的应用; 认为其在管理中, 贯穿培 训全程涉及到事先的沟通与交流、事中的提醒与反馈、事 后的跟踪与辅导、培训的统计与分析。辛雪[7]建立了生理 学微信公众平台, 并采用“问卷星”进行随堂测试及课后测 试。黄瑞滨等 $[8]$ 通过微信公众号推送理论学习内容, 并用 问卷星对学员进行每周测验, 评价并比较两组学员对本专 业理论知识掌握程度及对本科室住院医师规范化培训工 作的满意度。张震等[9]为将微课程、微课件、微学习“三 微”教学理念应用于气象职工在职培训, 提出基于微信公 众号的微信教学平台, 就平台的开发思路、功能实现以及 开发过程中的重难点进行阐述和分析, 探讨开发过程中存 在的问题, 并提出下一步的开发思路。郭琴 [10]结合 $5 \mathrm{~W}$ 模 式、营销理论、SWTO 等理论, 从传播主体、传播内容、 传播方式、传播对象、传播效果五个方面, 对三家培训机 构运用微信的策略进行分析。

\section{2. 移动互联网技术在中心培训管理中的应用}

为有效解决前文提到的培训项目管理流程中存在的 问题, 中心进行了探索和实践。

\subsection{1. 问卷平台的应用}

当前, 问卷平台的种类有很多种, 如问卷星、腾讯问 卷等。培训管理过程, 问卷平台的应用实践主要在接 (送) 信息登记、学员报名信息登记 (图4)、课程评估和项目 测评等工作。

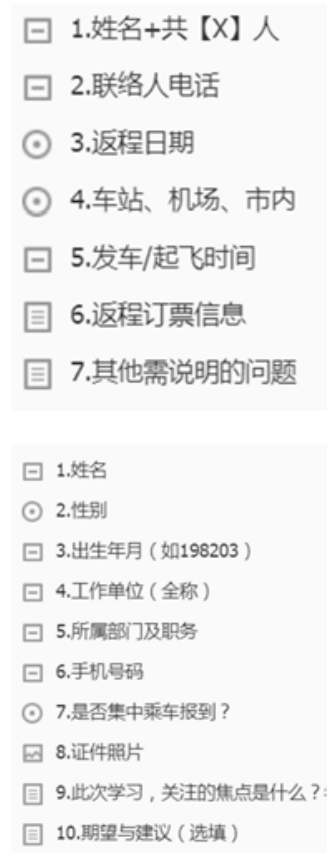

图5 接送信息(左)及学员报名信息问卷大纲(右)。 
在培训管理中, 学员报名数据整理的便捷性是考虑采 用问卷平台的最大因素。由于传统的学员报名登记过程贯 穿发文寄出到学员报到整个过程。为了更直观的研究学员 数据整理的便捷性, 我们对图2、图3所示的统计数据进行 了曲线拟合, 得到图6所示的学员报名过程变化趋势函数 图:
假设学员信息录入过程工作的时间消耗为常量 $A(A>0)$, 用离散函数 $N(t)$ 表示在第 $t$ 天 $\left(t \in N^{+}\right)$收到的学员 报名信息的数量，则第 $t$ 日完成报名登记工作的精力消耗 函数 $F(t)$ 为:

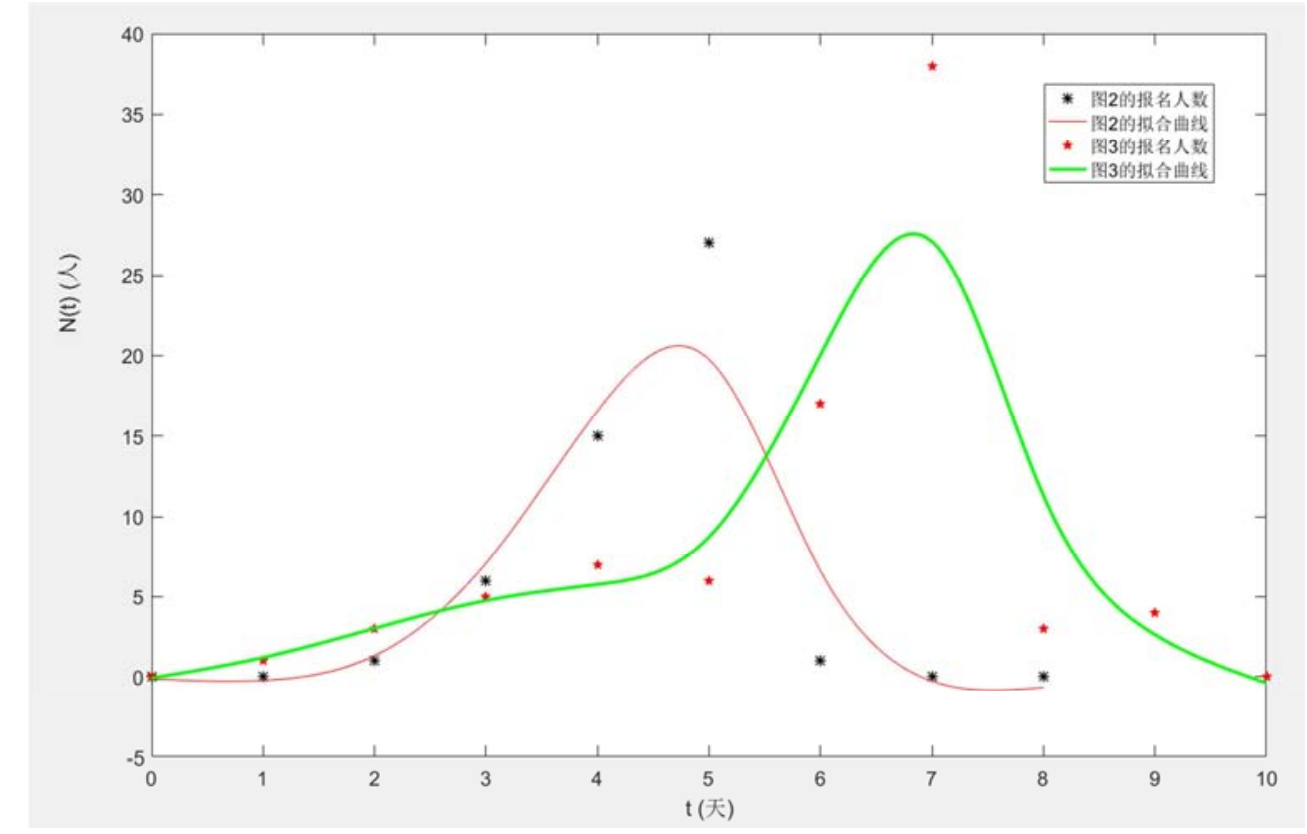

图6 对图2、图3学员报名趋势的曲线拟合。

$$
F(t)=A \cdot \sum_{i=0}^{t} N(i), \quad t \in\left(0, t_{\text {end }}\right]
$$

其中: $t_{\text {end }}$ 为发文寄出后到报到日的总天数。

而利用问卷平台进行学员报名信息采集时, 由于将信 息的录入交由学员或其所在单位操作，报名完成后仅需将 数据集中导出进行编辑处理即可, 因此, 在问卷平台辅助 下的学员报名信息工作是不连续的, 且工作量一般集中在 报到前的某几天的数十分钟。

相比之下, 基于问卷平台的学员报名信息统计的便捷 优势显而易见。

此外, 探索问卷平台在培训管理过程中的应用也考虑 了以下五方面的因素:

\section{(1) 数据存储安全性}

对于学员接 (送) 站信息及报名信息的统计一般都会 要求填写姓名、手机号码甚至是单位职务等敏感信息, 因 此对数据信息的安全性必须予以考虑。

问卷星和腾讯问卷等问卷平台, 采用多种方式和方法 保障用户提交数据和采集数据的安全性和提供加密服务, 尽力确保数据不会外泄, 且未经许可的情况下不会公开、 分享相关信息，不提供回收数据的共享合作。

(2) 数据管理的实时性

得益于互联网和智能手机的快速发展, 网络问卷平台 (以腾讯问卷为例) 采集的数据都可以通过网络实时查看, 并将相关问卷采集的查看权限授权给培训管理组其他成 员，便于随时了解学员报名情况。

(3) 信息采集的完整性
问卷平台一般可设置必选题和选做题两种形式。如果 是培训管理过程需要学员必须提供的信息, 则可通过设置 必选题对信息进行采集，当完成信息的提交后方可继续进 行后续操作，以确保信息采集的完整性。

(4) 采集内容的多样性

问卷平台一般提供文字、手机号码、日期、附件等多 种形式的题目选项格式设置, 可对学员联系方式、报到 (返 程）日期、发到站/起落地时间、详细信息登记表、培训 交流材料以及学员照片进行多种形式的数据进行有效性 核验和采集。

(5) 采集途径的适应性

问卷平台在分享采集接入方式时多数采用二维码和 网址相结合的方式，便于用户在电脑、平板及手机等多终 端以扫描二维码和网址录入等方式在线填报信息。

\subsection{2. 在线协作文档工具的应用}

当前, WPS云文档、腾讯文档、石墨文档、伙伴云表 格等各式各样的在线协作文档工具为培训管理过程中以 低廉、便捷的手段实现扫码签到并核对信息提供了可能。 中心在在线协作文档工具实践中主要对石墨文档和云表 格进行了分析、探索和对比。主要实践集中在：(1)基于在 线表格、表单的签到及信息核对工作; (2)基于在线文档的 培训班报到路线、注意事项、课程安排等信息的实时共享。

首要, 依旧考虑信息的安全性因素。在线协作文档同 样运用了多种技术保障在线信息的安全性。 
其次, 考虑可行性。由于在线文档提供了Excel等文 档工具的几乎所有常见功能, 因此结合VLOOKUP、 COUNTIF等函数功能可以实现学员签到、信息的核对以 及未到学员和新增学员的识别与登记功能。

最后，考虑便捷性。因为在线协作文档工具是基于 WEB的云端协作的互联网技术, 打开网页就能查看和填报, 在云端实时自动保存, 且提供表单功能（图7），因此其 在学员签到时能够以扫码形式完成多人同时签到, 且兼具 联系方式的采集核对功能。

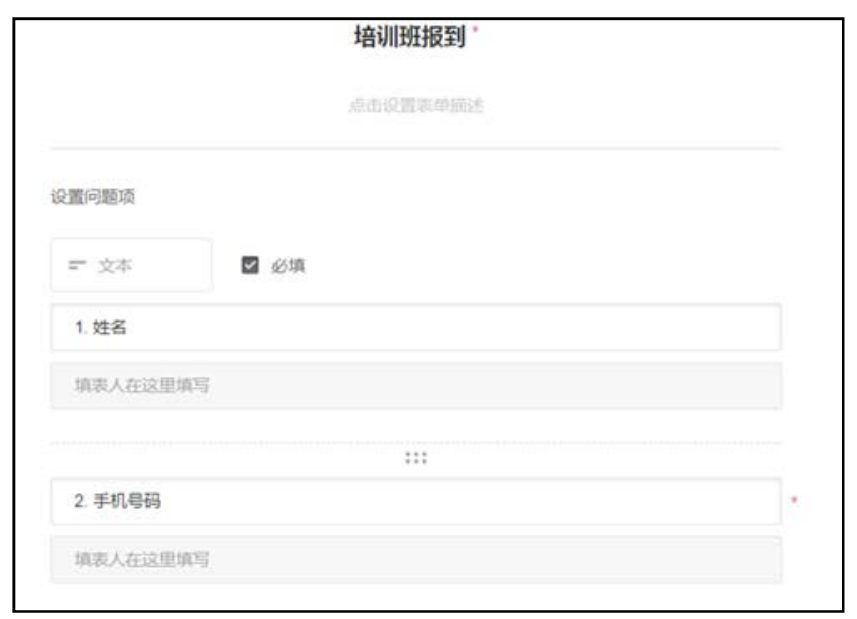

图7 在线协作文档工具的表单功能。

\subsection{3. 问卷平台与本地软件融合的应用}

图5 (右) 图中的学员报名信息问卷大纲中第 8 个题目 对学员照片进行了在线收集, 报名结束后可通过图8所示 流程图对学员照片进行重新命名处理, 得到符合要求的证 书申领名单和照片文件。

问卷平台与Excel融合的应用探索虽然能够实现高效 印制证书流程管理, 但是因其实现过程中需要多次调用 Excel函数并结合Windows操作命令, 因此并不适合所有人 运用, 且相对效率仍有待提高。为此, 中心尝试编写学员
证书照片的批量重命名软件程序的DEMO (如图9所示), 临时用于繁琐培训管理工作的处置。

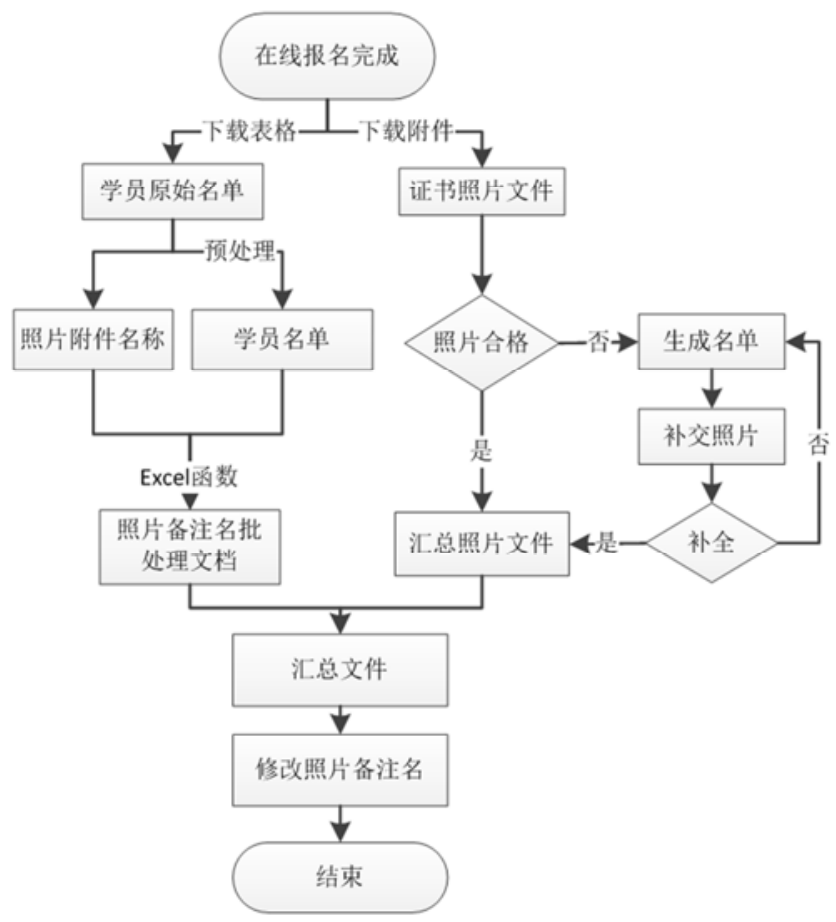

图8 问卷平台与Excel结合的证书管理流程。

软件 DEMO 能够基本实现以下功能:

(1)已交学员名单列表（含对应照片文件名称、文件大 小) ;

(2)已交照片但照片大小不合适的学员名单;

(3)照片文件名与名单不一致学员名单;

(4)未交照片的学员名单;

(5)学员照片备注名的批量修改;

(6)证书申领表数据自动生成。

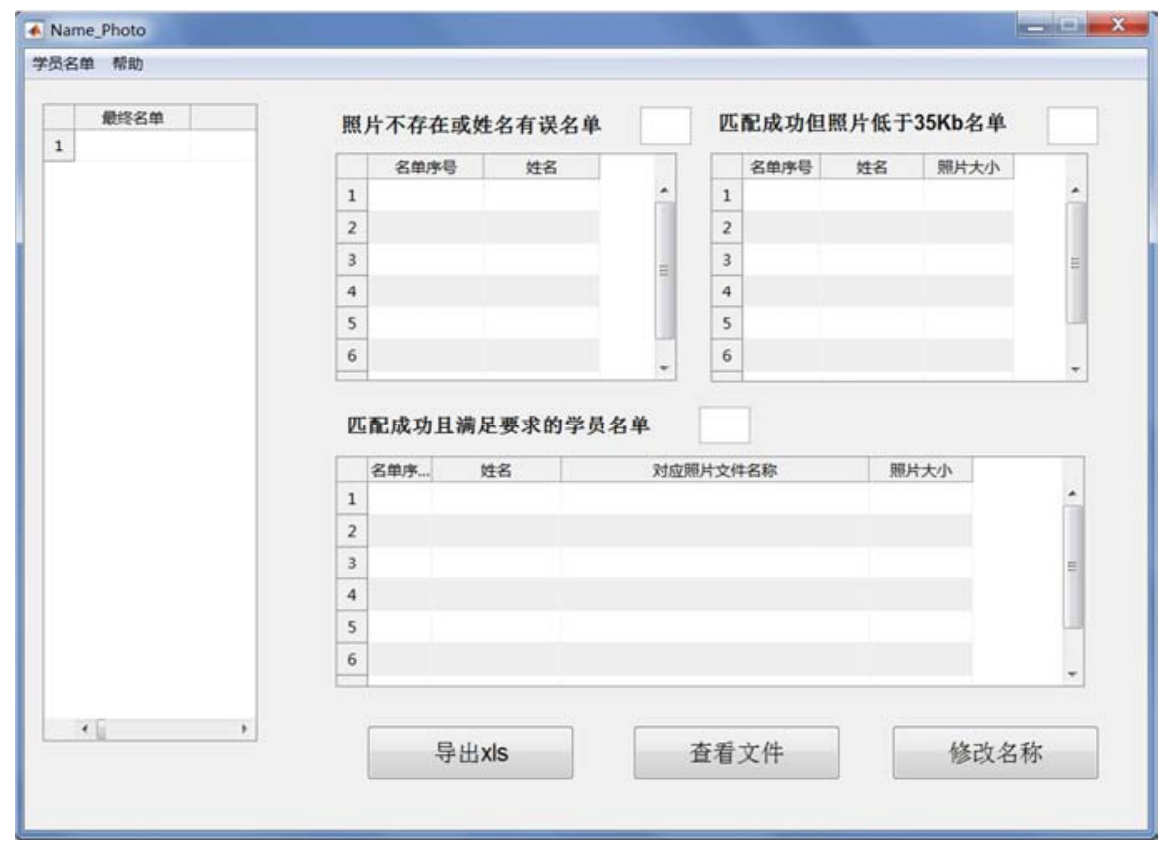




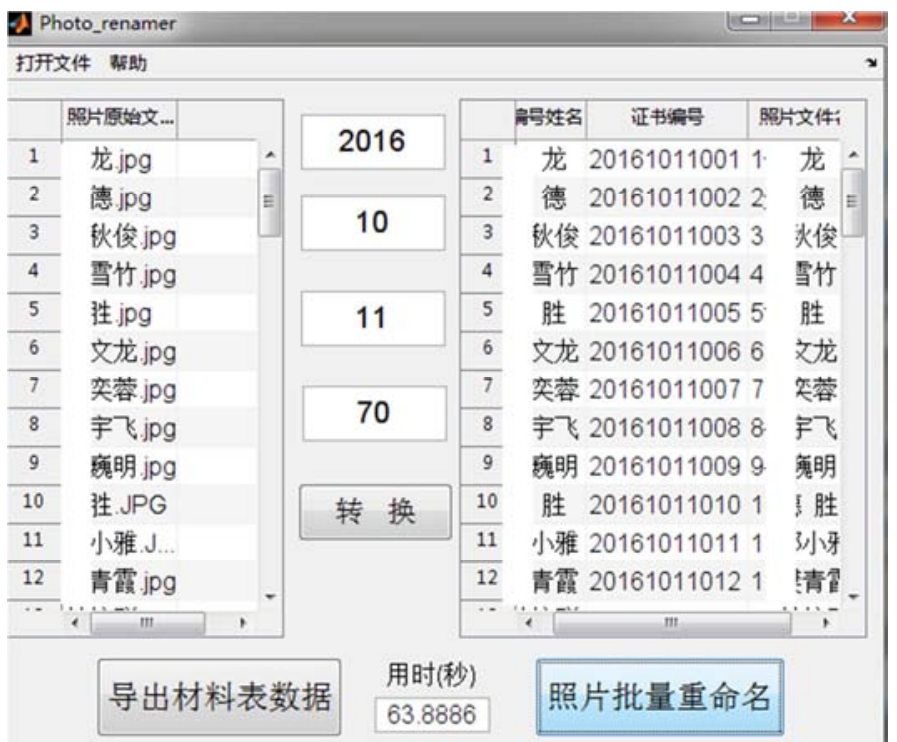

图9 基于程序软件DEMO的证书照片处理工具。

传统的证书照片处理流程是一个合理的时间段内, 单 调且有固定模式的重复工作, 若假设处理一个学员的照片 的平均时间为 $\bar{t}$, 且在该段时间内以同等的效率处理每个 照片文件, 则其 $n\left(n \in N^{+}\right)$位学员证书照片总共需要耗费 的时间为:

$$
T_{t r a}(n)=n \bar{t}
$$

经实验, $\bar{t}=9.05 s$ 。需要说明的是, $\bar{t}$ 的实验结果取 值因人而异。

对于问卷平台与Excel融合以及基于程序软件DEMO 工具的两种方式, 因其处理过程并非针对单个照片文件, 而是基于批量处理的思路进行的操作, 因此当学员数量 $T_{\text {demo }}(n)$ 时, 总共需要耗费的时间 $T_{x l s}(n) 、 T_{\text {demo }}(n)$ 才会随 着 $n$ 的增加而消耗更多的计算机软硬件资源而导致耗时
略微升高的趋势, 但是通常情况下由于学员数量基本不超 过 500 人, 因此当 $n \in(0,500]$ 时可视 $T_{x l s}(n) 、 T_{\text {demo }}(n)$ 近似 为常数函数, 主要与管理人员的操作熟练程度相关, 故有:

$$
\begin{gathered}
T_{x l s}(n)=\mu \cdot n^{0} \\
T_{\text {demo }}(n)=\delta \cdot n^{0}
\end{gathered}
$$

其中, $\mu>0, \delta>0, n \in(0,500]$ 且 $n \in N^{+}$。经实验得出 $\mu=71 s 、 \delta=31 s 。$

因此三种方式分别需要耗费的时间函 数曲线如图 10 所示。从图中可以看出, 传统的证书照片文件处理的时间 随着学员数量的增加而增加, 且当学员数量多于 8 人时, 其处理证书照片所需时间即超过其他两种方式。

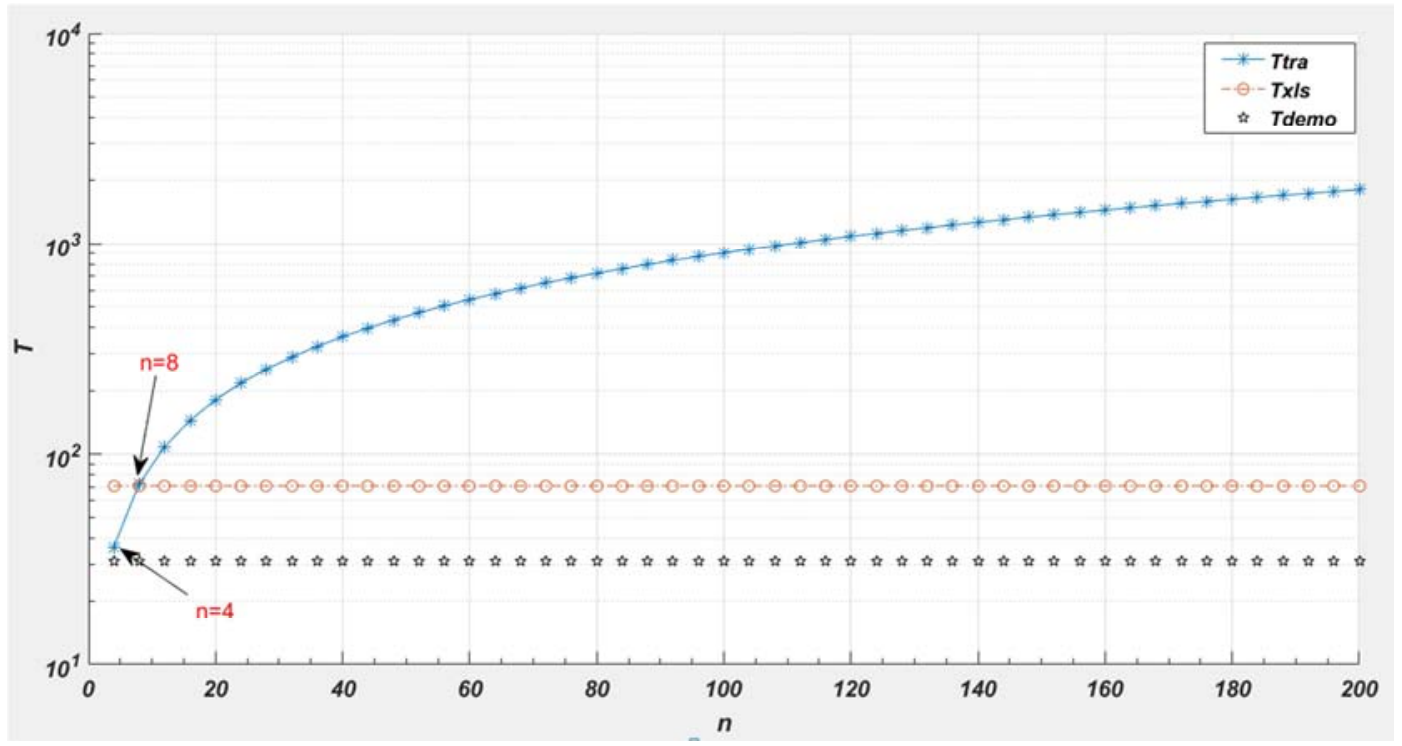

图10 三种方式处理照片文件需要耗费的时间函数曲线。 


\section{4. 中心开展微信公众平台培训管理板块建设的 思考}

2012年 8 月 18 日, 腾讯在微信的基础上新增功能模块, 推出了微信公众平台。微信公众平台依托微信, 不仅具有 其普适功能, 而且支持多种形式的外接拓展功能, 基于微 信消息高效抵达、口碑效应以及强交互氛围特征[11], 利 用培训管理的线上线下沟通交流增强公众号对学员的吸 引力和传播力度, 将会进一步增强公众号在培训管理乃至 科研咨询和教材推广中工作开展的便利性和普及性。

\section{1. 微信公众平台的普适功能}

通过微信公众平台，个人和企业、事业单位都可以向 特定群体发送文字、图片、语音、视频等内容, 进行全方 面的沟通与互动, 其主要功能定位有群发推送、自动回复 和一对一交流。

\section{2. 微信公众平台的拓展功能}

因为基于问卷平台和在线协作文档工具均属于移动 互联网技术, 可通过二维码及网址形式分享给群体成员。 因此结合微信公众平台的普适功能, 微信公众平台、问卷 平台及在线协作文档工具将能够在培训项目管理过程中 发挥巨大作用。

\section{3. 微信公众平台与培训管理}

根据培训管理的三个阶段的流程工作，结合微信公众 平台的普适功能和拓展功能, 在前期实践基础上, 得到图 11所示的培训管理与公众平台的关联图谱, 简单说明了各 功能在培训管理各阶段的应用场景。

\section{5. 结论}

在如今互联网技术发展日新月异的环境下, 管理效率 和管理方式的创新决定着生产力是否能够得到提升。培训 管理中传统的管理方式对人力、物力和精力的耗费, 逐步 不再适应新时代对于精细化管理的要求。

目前, 信息化系统和工具的重要性早已被培训管理人 员意识到, 但是由于软件（APP）、Web应用等工具的开 发周期长、投入资金多、功能不完善, 并不能有效在培训 管理中得到广泛应用。而高效的培训管理工具和手段的应 用又迫在眉睫。免费的微信及微信公众平台功能的不断开 发和完善, 吸引了越来越多的人使用微信并关注微信公众 平台的内容, 并为信息的高效和广泛传播提供了良好途径。 因此, 以微信及微信公众平台为主、混合多种移动互联网 技术的培训管理工具, 为培训管理方式方法的创新提供了 无限可能, 如果能合理设计、深入挖掘、积极探索各种移 动互联网技术的综合运用, 做好平台用户的粘性和活跃性, 必将促使培训管理工作更加便捷、高效。

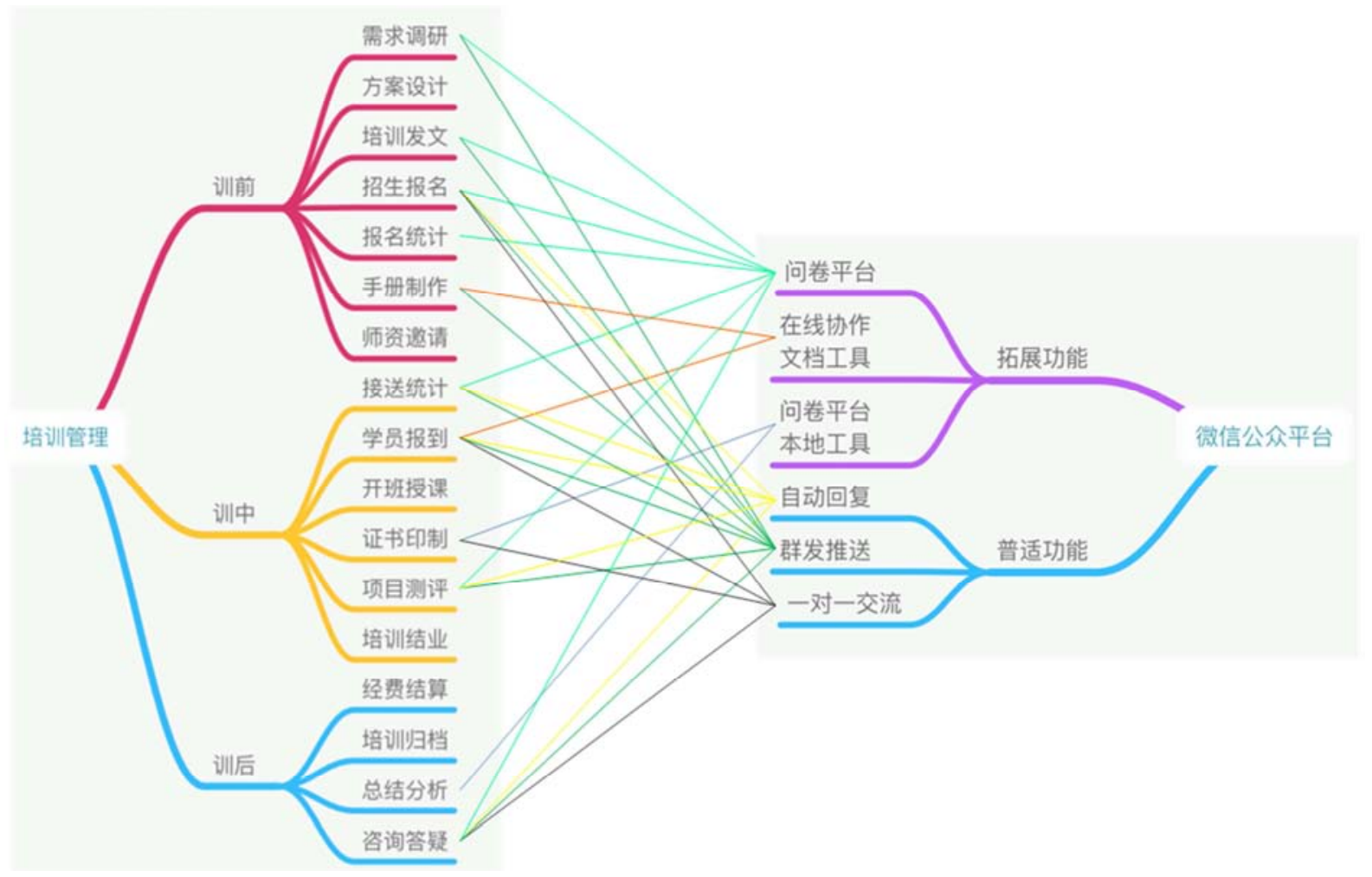

图11 培训管理与公众平台的关联图谱。 


\section{参考文献}

[1] 洪东. 某事业单位干部培训管理系统的设计与实现[D]. 厦 门大学, 2014 .

[2] 李雪玲. 干部培训管理信息系统的分析与设计 $[\mathrm{J}]$.电脑编程 技巧与维护,2016,(2):55-57.

[3] 间琳. 领导干部培训管理流程建设 [J]. 继 续教 育,2011,25(09):16-17.

[4] 移动互联网技术呈现多样化趋势[J].硅谷,2012(09):16-17.

[5] 郭伟才.浅析微信技术在培训管理中的应用[J]. 科技经济导 刊,2016(22):26.
[6] 余雅斌. 微信及公众平台嵌入教师培训的应用探索[J]. 高教 论坛,2014(09):97-99.

[7] 辛雪,纳仁高娃. “微信公众平台+问卷星”在生理学教学中的 应用[J].医学教育研究与实践,2017,25(03):428-430.

[8] 黄瑞滨,马焕杰,许伟雄, 黄旭璇,陈小丽, 刘源- 《继续医学教 育》2018年11期.郭琴. 微信在教育培训机构中的运用策略 分析[D].上海师范大学,2016.

[9] 郭琴. 微信在教育培训机构中的运用策略分析[D].上海师 范大学,2016.

[10] 王艳玲,李宸.微信公众号的传播特性及其营销优势 [J].新闻 爱好者,2017(10):39-41.

[11] 张震,王文玉.基于微信公众号的微信教学平台研究与开发 [J].时代教育,2016,(21):231-232,23. 